\title{
Sagittal and vertical craniofacial growth and attainment of circumpubertal middle phalanx maturation (MPM) stages: A multiple regression study
}

\author{
Perinetti, Giuseppe *; Sverko, Davide **; Dal Borgo, Beatrice **; Contardo, Luca ** \\ * Private practice, Nocciano (PE), Italy. \\ ** Department of Medical, Surgical and Health Sciences, School of Dentistry, University of Trieste, Trieste, Italy
}

\begin{abstract}
Introduction: Orthodontic treatment for most of the skeletal malocclusions have specific optimal timing according to the skeletal maturation phases. The knowledge of whether attainment of a specific maturation phase is associated with the different sagittal and vertical craniofacial growth pattern then becomes of clinical relevance.

Aim: This cross-sectional study evaluated whether sagittal and vertical craniofacial growth pattern, has an association with the age of attainment of the circumpubertal skeletal maturation phases.

Materials and methods: A total of 300 subjects (170 females and 130 males) were included in the study (mean age, $12.0 \pm 1.5$ years; range, 8.315.6 years). These subjects were equally distributed in the circumpubertal middle phalanx maturation (MPM) stages 2,3 and 4 . Subsequently, multiple regression models were run for each MPM stage group to assess the significance of the association of cephalometric parameters (SNA, SNB, ANB, PP/MP, CoGoMe, SN/MP and NSBa angles) with age of attainment of the corresponding MPM stage (in months).

Results: Only sex yielded significant associations, with females having anticipated attainment of each of the circumpubertal MPM stage. Conclusion: These results show no significant correlations of the different sagittal and vertical cephalometric parameters with the age of attainment of the circumpubertal skeletal maturation phases.
\end{abstract}

Perinetti G, Sverko D, Dal Borgo B, Contardo L. Sagittal and vertical craniofacial growth and attainment of circumpubertal middle phalanx maturation (MPM) stages: A multiple regression study. South Eur J Orthod Dentofac Res. 2018;5(1):12-17.

Submitted: January 30, 2018; Revised: February 22, 2018; Published: April 30, 2018

\section{INTRODUCTION}

Different types of skeletal malocclusions require optimal timing of intervention, i.e., a given skeletal maturation phase. ${ }^{1,2}$ Therefore, the knowledge of whether attainment of a specific maturation phase is associated to the different sagittal and vertical craniofacial growth pattern becomes of relevance in dentofacial orthopedics and orthodontics. For instance, deficiency ${ }^{3}$ and increased ${ }^{4}$ mandibular length in skeletal Class II and Class III subjects, respectively, was suggested to be in part a consequence of the different durations of the pubertal growth spurt in these

Corresponding Author:

Perinetti Giuseppe

Via San Lorenzo 69/1,

65010 Nocciano (PE), Italy.

e-mail: G.Perinetti@yahoo.com subjects, as compared to that of Class I subjects. ${ }^{5-8}$ However, in spite of the wide indications for orthopedic treatments in case of skeletal malocclusion, only a few studies investigated on possible correlation between craniofacial growth pattern and attainment/duration of the different skeletal maturation phases ${ }^{5-7,9,10}$ (according to which timing of intervention is based ${ }^{1,2}$ ). These studies also reported contrasting evidence with clinically relevant ${ }^{5-9}$ or not relevant ${ }^{10}$ correlations.

All of these previous investigations ${ }^{5-10}$ used the cervical vertebral maturation (CVM) method ${ }^{2,11}$ to assess the attainment of the different circumpubertal skeletal maturation phases. However, the different variants of the method, ${ }^{12-15}$ and the contrasting evidence regarding the reliability of the CVM method in detecting the mandibular growth peak ${ }^{13,14,16-18}$ or repeatability ${ }^{19}$ would limit the external validity of the method. 
As an alternative to the hand and wrist maturation ${ }^{20}$ and CVM ${ }^{2,11}$ methods, a recent 5-stage third finger middle phalanx maturation (MPM) method has been proposed ${ }^{21}$ as a radiographical indicator of skeletal maturation phase. This method would have a satisfactory diagnostic capability in detecting the mandibular growth peak ${ }^{21}$ and has the advantage of being minimally invasive, allowing repetition of the recordings for a close monitoring of the ossification events, i.e. passage from one stage to the subsequent. ${ }^{22}$

Therefore, using multivariate models, this cross-sectional study evaluated whether sagittal and vertical craniofacial growth pattern has an association with the age of attainment of the circumpubertal skeletal maturation phases according to the MPM method.

\section{MATERIALS AND METHODS}

Study population and design

The database between January 2008 and July 2017 of the Sections of Stomatology of the Department of Medical, Surgical and Health Sciences, University of Trieste, was screened. This study included subjects who were seeking orthodontic treatment and who had never been treated before. As a routine procedure, a signed informed consent for releasing diagnostic material for scientific purposes was obtained from the patients' parents prior to entry into treatment, procedures followed adhered to the World Medical Organization Declaration of Helsinki ${ }^{23}$ and the protocol was reviewed and approved by the local Ethical Committee. In particular, in the first clinical session a lateral cephalograms were taken as a part of the pre-treatment clinical recording. The following inclusion criteria were applied: i) age between 7 and 17 years; ii) circumpubertal skeletal maturation between MPM stage 2 and 4; iii) absence of any craniofacial anomaly, or extensive dental caries or restorations; iv) good general health; v) no history of trauma at the craniofacial region; and vi) Caucasian ethnicity. A dedicated X-ray machine (KODAK 8000C; Eastman Kodak Company) was employed for the recording of lateral head cephalograms. Settings were of 73-77 kV, $12 \mathrm{~mA}$ with an exposure time of 0.80 seconds. Images were saved at $300 \mathrm{dpi}$ resolution and radiographs of low quality were excluded. An experienced orthodontist (BDB) assisted by a second operator (DS) screened the cases for inclusion. A further experienced orthodontist (LC) was involved to ensure correct enrollment and, in case of disagreement, discussion was made until satisfaction of both operators. From an initial sample of over 450 subjects, total of 300 subjects (170 females and 130 males) were included in the study (mean age, 12.0 \pm 1.5 years; range, $8.3-15.6$ years).

\section{Cephalometric analysis of the face and cervical vertebrae}

A customized digitization regimen and analysis with cephalo- metric software (Viewbox, version 3.0, dHAL Software, Kifissia, Greece) was used for all cephalograms examined in this study. The cephalometric analysis of the face required the digitization of 10 landmarks (Figure 1).24 The customized cephalometric analysis included 7 angular measurements as follows (Figure 1): maxillary prognathism (SNA angle), mandibular prognathism (SNB angle), maxillo-mandibular relationship (ANB angle), palatal inclination relative to the mandibular plane (PP/MP angle), mandibular inclination relative to the cranial base ( $\mathrm{SN} /$ MP angle), condylar angle (CoGoMe angle) and cranial base angle (NSBa angle). All of the cephalograms were traced by one investigator (DS), and a second investigator (BDB) checked each tracing for accuracy.

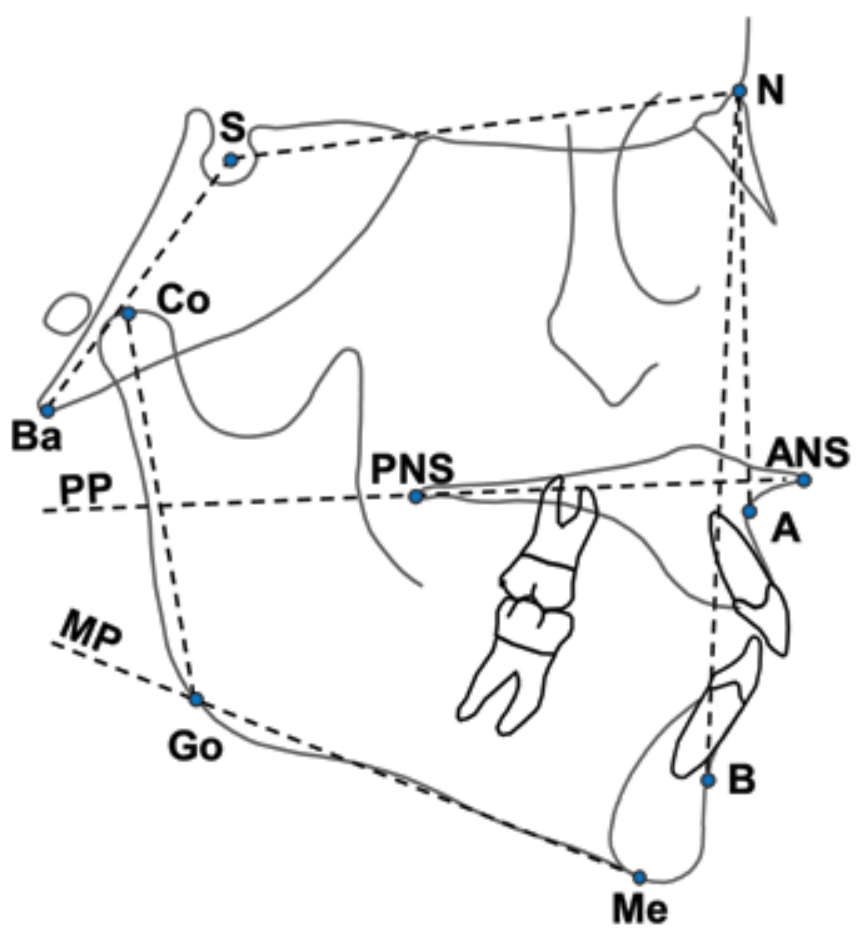

Figure 1. Diagram of the cephalometric measurements of the craniofacial complex. Landmarks: $A$, subspinale; $B$, supramentale; $N$, nasion; $S$, centre of the sella turcica; Ba, Basion; Co, Condylion; Go, Gonion; Me, menton; ANS, anterior nasal spine; PNS, posterior nasal spine; Planes: PP, palatal plane; MP, mandibular plane. See text for details.

\section{Middle phalanx maturation (MPM) assessment}

The MPM method used herein comprises 5 stages as previously reported ${ }^{21}$ and briefly defined as follows (Figure 2): A) MPM stage 1 , when the epiphysis is narrower than the metaphysis, or when epiphysis is as wide as metaphysis but with both tapered and rounded lateral borders. Epiphysis and metaphysis are not fused; B) MPM stage 2, when the epiphysis is at least as wide as the metaphysis with sides increasing thickness and showing a 


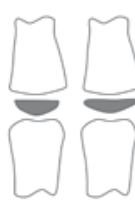

MPS1

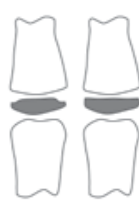

MPS2

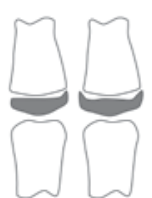

MPS3

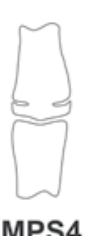

MPS4

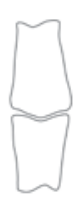

MPS5
Figure 2. Diagram of the third finger middle phalanx maturation stages. See text for details.

clear line of demarcation at right angle, either with or without lateral steps on the upper contour. In case of asymmetry between the two sides, the more mature side is used to assign the stage; C) MPM stage 3, when the epiphysis is either as wide as or wider than the metaphysis with lateral sides showing an initial capping towards the metaphysis. In case of asymmetry between the two sides, the more mature side is used to assign the stage. Epiphysis and metaphysis are not fused; D) MPM stage 4, when the epiphysis begins to fuse with the metaphysis although contour of the former is still clearly recognizable. Both sides of the epiphysis form obtuse angle to distal border, and the capping may still be detectable; E) MPM stage 5, when the epiphysis are totally fused with the metaphysis.

\section{Method error}

With the aim of quantifying the full method error of the recordings for each recorded parameter, the method of moments variance estimator ${ }^{25}$ was used on a random sample of 20 replicate measurements. Therefore, the mean error and 95\% confidence intervals (CIs) between the repeated recordings were calculated using the MME variance estimator. Moreover, the repeatability in the CVM stage assignment in the same pairs of measurements was evaluated using the percentage of agreement, and by both unweighted and linear weighted kappa coefficients presented as mean and 95\% CI. The kappa coefficient ranges from zero for no agreement to 1 for perfect agreement. ${ }^{26}$

\section{Statistical analysis}

The SPSS software version 20 (SPSS ${ }^{\bullet}$ Inc., Chicago, Illinois, USA) was used to perform the subsequent data analysis. After testing the normality of the data with the Shapiro-Wilk test and Q-Q normality plots of the residuals, and the equality of variance among the datasets using a Levene test, parametric methods were used for data analysis. ${ }^{27}$ The significance of the difference in each craniofacial and cervical vertebral cephalometric parameter among the MPM stage groups was evaluated through one-way analysis of variance. ${ }^{27}$
Moreover, within each MPM stage group, the association of each of the craniofacial parameters (explanatory variables) with the chronological age in months (dependent variable) was investigated using multiple linear regressions. In particular, a bivariate correlation matrix with Pearson coefficient was executed for each MPM stage group including all the craniofacial cephalometric parameters, according to which the $\mathrm{SNB}$ and PP/MP angles were excluded from the multivariate models in which were entered instead: sex (male), SNA angle, ANB angle, SN/MP angle, CoGoMe angle and NSBa angle. For each multiple regression model, multi-co-linearity among the remaining explanatory variables was also again checked for though the tolerance and variance inflation factor parameters.

A p $<0.05$ was used for rejection of the null hypothesis.

\section{RESULTS}

For the face measurements, greatest method error of $1.16^{\circ}$ (0.90-1.61) was for the CoGoMe angle. The overall percentage of agreement for the MPM stages was $92 \%$ (23 cases out of 25 ). The unweighted kappa coefficient was 0.88 (0.71-1), and the weighted kappa coefficient was 0.90 (0.78-1).

Chronological ages for each group according to the sexes are reported in Table 1. For females, mean ages were 10.9, 11.4 and 12.5 years in the MPM stage groups 2, 3 and 4, respectively. For males, mean ages were 11.7, 12.9 and 13.3 years in the MPM stage groups 2, 3 and 4, respectively. The difference between the sexes within each group was significant $(\mathrm{p}=0.003$, at least).

Descriptive statistics for each analyzed parameter is reported in Table 2. The SNA angle ranged from $80.1^{\circ} \pm 4.0$ (MPM stage 2) to $80.8^{\circ} \pm 3.6$ (MPM stage 3 ); the SNB angle ranged from $76.8^{\circ} \pm 3.5$ (MPM stage 3 ) to $76.9^{\circ} \pm 3.5$ (CVM stage 3 ); the ANB angle ranged from $3.4^{\circ} \pm 2.3$ (MPM stage 2) to $4.0^{\circ}$ $\pm 1.9^{\circ}$ (MPM stage 3 ); the PP/MP angle ranged from $24.9^{\circ} \pm 5.4$ (MPM stage 2) to $26.0^{\circ} \pm 5.5$ (CVM stage 4 ); the SN/MP angle ranged from $33.0^{\circ} \pm 5.6$ (MPM stage 2) to $33.8^{\circ} \pm 6.3$ (MPM stage 4); the CoGoMe angle ranged from $123.2^{\circ} \pm 5.7$ (MPM stage 2) to $124.1^{\circ} \pm 6.2$ (MPM stage 4 ); the NSBa angle ranged from $123.2^{\circ} \pm 5.7$ (MPM stage 2) to $130.0^{\circ} \pm 4.7$ (MPM stage 4). For all of these craniofacial cephalometric parameters the differences among the groups were not statistically significant.

Results of the multiple linear regression models according to each MPM stage group are reported in Table 3. In each model. Only the sex yielded a significant association with the age of attainment of the corresponding MPM stage. In particular, in the MPM stage 2 group (Model 1 ) the $\mathrm{R}^{2}$ was of 0.113 with the sex (male) with $\beta$ coefficient of 8.42 ( $\mathrm{p}=0.005)$; in the MPM stage 3 group (Model 2 ) the $\mathrm{R}^{2}$ was of 0.156 with the sex (male) with $\beta$ coefficient of $16.22(\mathrm{p}=0.000)$; in the MPM stage 4 group (Model 3) the $\mathrm{R}^{2}$ was of 0.052 with the sex (male) with $\beta$ coefficient of $9.43(\mathrm{p}=0.002)$. 


\section{DISCUSSION}

Through multivariate models, the present study showed no significant correlations of the different sagittal and vertical cephalometric parameters with the age of attainment of each of the circumpubertal MPM stages 2, 3 and 4. The only significant association was with sex, where females had anticipated attainment of each MPM stage as compared to males.

The knowledge of the associations between the timing of skeletal maturation and craniofacial growth is of primary importance when planning a functional treatment for most of the skeletal malocclusions, including those on the sagittal ${ }^{2,28,29}$ and vertical dimensions. ${ }^{30,31}$ In particular, optimal timing for functional/ orthopedic treatment for skeletal Class II and Class III has been reported to be late ${ }^{32-34}$ and early, ${ }^{2}$ respectively.

For the first time, the present study used the MPM method, which has the advantage of easy interpretation and minimal radiation exposure. ${ }^{1}$ Moreover, the MPM stages 2 and 3 have been associated with the onset and maximum mandibular growth peak, respectively, in most of the subjects. ${ }^{21}$ Intersubject variability in the duration of each stage of the different radiographical indicator of skeletal maturity has been reported, ${ }^{35}$ including the MPM method. ${ }^{21}$ Even though this aspect would have major implications when dealing with induvial subjects, results obtained by correlation analyses in a group of subjects would be less affected by such a limitation.

In the present study, earlier attainment of each of the circumpubertal MPM stages for females as compared to males has been seen (Table 1). This evidence has also been confirmed by the results of the multivariate models where $\beta$ coefficients ranged between 8.43 and 16.22 (Table 3). Interestingly, the greatest difference between sexes was seen for the age of attainment of the MPM stage 3 when mandibular growth peak has been reported to occur. ${ }^{21}$ This evidence is in accordance with previous investigations on different samples using the MPM, ${ }^{21} \mathrm{CVM}^{2,35,36}$ or HWM ${ }^{20,37,38}$ methods.

Table 1. Chronological age for each middle phalanx maturation (MPM) stage according to the sexes.

Each MPM stage group includes equal number of females and males. Data on age are presented as mean $\pm S D$. Diff., significance of the difference between the sexes within each MPM stage group. S, statistically significant.

\begin{tabular}{cccc}
\hline \multirow{2}{*}{ Sex } & \multicolumn{3}{c}{ Middle phalanx maturation stage group } \\
\cline { 2 - 4 } & $\begin{array}{c}\text { MPM stage 2 } \\
(\mathbf{N}=\mathbf{1 0 0})\end{array}$ & $\begin{array}{c}\text { MPM stage 3 } \\
(\mathbf{N}=\mathbf{1 0 0})\end{array}$ & $\begin{array}{c}\text { MPM stage 4 } \\
(\mathbf{N}=\mathbf{1 0 0})\end{array}$ \\
\hline Females & $10.9 \pm 1.3$ & $11.4 \pm 1.4$ & $12.5 \pm 1.1$ \\
\hline Males & $11.7 \pm 1.1$ & $12.9 \pm 1.4$ & $13.3 \pm 1.3$ \\
\hline Diff. & $0.003 ; \mathrm{S}$ & $0.000 ; \mathrm{S}$ & $0.001 ; \mathrm{S}$ \\
\hline
\end{tabular}

Table 2. Descriptive statistics for the craniofacial parameters (in degrees) for each group.

Each MPM stage group includes equal number of females and males. Data are presented as mean $\pm S D$. Diff., significance of the Levels of differences among the MPM stage groups for each cephalometric parameter. NS, not statistically significant.

\begin{tabular}{ccccc}
\hline \multirow{2}{*}{$\begin{array}{c}\text { Parameter } \\
(\text { degree })\end{array}$} & $\begin{array}{c}\text { MPM stage } \mathbf{2} \\
(\mathbf{N}=\mathbf{1 0 0})\end{array}$ & $\begin{array}{c}\text { MPM stage } 3 \\
(\mathbf{N}=\mathbf{1 0 0})\end{array}$ & $\begin{array}{c}\text { MPM stage } \mathbf{4} \\
(\mathbf{N}=\mathbf{1 0 0})\end{array}$ & Diff. \\
\cline { 2 - 4 } & $80.1 \pm 4.0$ & $80.8 \pm 3.6$ & $80.3 \pm 3.6$ & $0.365 ; \mathrm{NS}$ \\
\hline SNA angle & $76.8 \pm 3.9$ & $76.8 \pm 3.5$ & $76.9 \pm 3.9$ & $0.971 ; \mathrm{NS}$ \\
\hline SNB angle & $3.4 \pm 2.3$ & $4.0 \pm 1.9$ & $3.4 \pm 2.7$ & 0.058 ; NS \\
\hline ANB angle & $24.9 \pm 5.4$ & $25.3 \pm 5.1$ & $26.0 \pm 5.5$ & $0.312 ; \mathrm{NS}$ \\
\hline PP/MP angle & $33.0 \pm 5.6$ & $33.4 \pm 6.1$ & $33.8 \pm 6.3$ & $0.690 ; \mathrm{NS}$ \\
\hline SN/MP angle & $123.2 \pm 5.7$ & $123.7 \pm 5.9$ & $124.1 \pm 6.2$ & $0.608 ; \mathrm{NS}$ \\
\hline CoGoMe angle & $127.8 \pm 10.6$ & $128.9 \pm 4.8$ & $130.0 \pm 4.7$ & $0.112 ; \mathrm{NS}$ \\
\hline NSBa angle & & & & \\
\hline
\end{tabular}

Table 3. Results of the backward multiple linear regressions for the association of craniofacial cephalometric parameters with the chronological age (in months) for each MPM stage.

Independent variables entered in each model: sex, SNA angle, ANB angle, SNIMP angle, CoGoMe angle and NSBa angle. Results of the multiple linear regressions are presented as $\beta$ (SE); R2, coefficient of determination. Sig., Level of significance; $S$, statistically significant; $N S$, not statistically significant.

\begin{tabular}{|c|c|c|c|}
\hline Parameter (degree) & $\beta(\mathrm{SE})$ & $\mathbf{t}$ & Sig. \\
\hline \multicolumn{4}{|c|}{ Model 1: Age of attainment of MPM stage $2(N=100), R^{2}=0.113$} \\
\hline Sex (male) & $8.42(2.96)$ & 2.846 & $0.005 ; S$ \\
\hline SNA angle & $-0.30(0.64)$ & -0.468 & 0.641 ; NS \\
\hline ANB angle & $1.75(0.90)$ & 1.943 & $0.055 ; \mathrm{NS}$ \\
\hline $\mathrm{MP} / \mathrm{SN}$ angle & $-1.00(0.54)$ & -1.847 & $0.068 ; \mathrm{NS}$ \\
\hline CoGoMe angle & $0.56(0.41)$ & 1.346 & 0.182 ; NS \\
\hline NSBa angle & $0.14(0.14)$ & 1.346 & 0.182 ; NS \\
\hline
\end{tabular}

Model 2: Age of attainment of MPM stage $3(N=100), R^{2}=0.156$

\begin{tabular}{llll}
\hline Sex (male) & $16.22(3.61)$ & 4.493 & $0.000 ; \mathrm{S}$ \\
\hline SNA angle & $0.17(0.74)$ & 0.227 & $0.821 ; \mathrm{NS}$ \\
\hline ANB angle & $0.02(1.18)$ & 0.016 & $0.988 ; \mathrm{NS}$ \\
\hline MP/SN angle & $-0.24(0.58)$ & -0.406 & $0.685 ; \mathrm{NS}$ \\
\hline CoGoMe angle & $0.36(0.48)$ & 0.739 & $0.462 ; \mathrm{NS}$ \\
\hline NSBa angle & $0.37(0.44)$ & 0.847 & $0.399 ; \mathrm{NS}$ \\
\hline
\end{tabular}

\begin{tabular}{|c|c|c|c|}
\hline Sex (male) & $9.43(3.00)$ & 3.144 & $0.002 ; \mathrm{S}$ \\
\hline SNA angle & $0.06(0.67)$ & 0.088 & 0.930 ; NS \\
\hline ANB angle & $-0.12(0.80)$ & -0.152 & 0.879 ; NS \\
\hline $\mathrm{MP} / \mathrm{SN}$ angle & $0.22(0.49)$ & 0.440 & 0.661 ; NS \\
\hline CoGoMe angle & $-0.29(0.40)$ & -0.718 & $0.474 ; \mathrm{NS}$ \\
\hline NSBa angle & $0.01(0.38)$ & 0.008 & $0.999 ; \mathrm{NS}$ \\
\hline
\end{tabular}


In the present study, no significant differences in any of the cephalometric parameter was seen among the different MPM stage groups (Table 2) and none of these craniofacial parameters was significantly associated with the age of attainment of each of the investigated MPM stage (Table 3). To date very little research has focused on the possible association between the timing of the circumpubertal skeletal maturation phases and sagittal craniofacial growth. ${ }^{5-10}$ In this regard, a shorter and longer pubertal growth spurt, as recorded through the ages of attainment of CVM stages 3 and 4, have been reported for untreated skeletal Class II ${ }^{7,9}$ and Class III ${ }^{6,8,9}$ subjects, respectively. On the contrary, no significant association has been reported between the sagittal craniofacial growth pattern and attainment of the circumpubertal CVM stages 2, 3 and 4. 5,10 In a study, ${ }^{6}$ the average age at onset of the pubertal peak was similar for both skeletal Class I and Class III subjects, although Class III subjects exhibited a prolonged pubertal CVM stage 3. The use of multivariate models, ${ }^{5,10}$ instead of univariate analyses, ${ }^{6-9}$ may explain apparent inconsistencies among the studies. Indeed, the two investigations ${ }^{5,10}$ that failed to detect significant associations used multivariate models. Although one ${ }^{5}$ of these studies missed the reporting of data regarding vertical growth, and this parameter was not used for adjustments in the multiple regression model. However, for skeletal Class II and Class III subjects, the entity of malocclusion has to be taken into account, and further studies may include subjects with severe skeletal sagittal malocclusion.

Only a previous study ${ }^{10}$ investigated on possible associations between vertical craniofacial growth and timing of attainment of skeletal maturation phases. This study ${ }^{10}$ was based on the CVM method and reported that greater the MP/SN angle and lower the age for the attainment of the CVM stage 3. In particular, with unitary increments in SN/MP accounted for about 0.7 months anticipation of the attainment of the CVM stage 3. Even though statistically significant, such correlation would have little clinical relevance being limited to extreme cases. With this exception, no other significant correlations were reported. The present results may be considered consistent with this previous investigation. ${ }^{10}$

Taking together sagittal and vertical craniofacial growth pattern, the overall $\mathrm{R}^{2}$ retrieved for the different models were low as $0.113,0.156$ and 0.052 for the MPM stages 2, 3 and 4, respectively (Table 3). Therefore, other potential factors would contribute to the age of the attainment of the skeletal maturation phase. These factors may include genetics, ethnicity, nutrition and socioeconomic status. ${ }^{39,40}$

The present study thus warrants further investigations using different growth indicators, such as standing height under longitudinal designs, which can take into account potential effects by confounding factors. The present results apply to Caucasian subjects.

\section{CONCLUSIONS}

Age variations in the attainment of the different circumpubertal MPM stages 2, 3 and 4 have been seen to be independent of both the sagittal and vertical craniofacial growth pattern.

\section{CONFLICT OF INTEREST}

The authors declare that there is no conflict of interest regarding the publication of this paper. 


\section{REFERENCES}

1. Perinetti G, Contardo L. Reliability of Growth Indicators and Efficiency of Functional Treatment for Skeletal Class II Malocclusion: Current Evidence and Controversies. Biomed Res Int. 2017;2017:1367691.

2. Baccetti T, Franchi L, McNamara JA, Jr. The cervical vertebral maturation (CVM) method for the assessment of optimal treatment timing in dentofacial orthopedics. Semin Orthod 2005;11(3):119-29.

3. Stahl F, Baccetti T, Franchi L, McNamara JA, Jr. Longitudinal growth changes in untreated subjects with Class II Division 1 malocclusion. Am J Orthod Dentofacial Orthop. 2008;134(1):125-37.

4. Reyes BC, Baccetti T, McNamara JA, Jr. An estimate of craniofacial growth in Class III malocclusion. Angle Orthod. 2006;76(4):577-84.

5. Armond MC, Generoso R, Falci SG, Ramos-Jorge ML, Marques LS. Skeletal maturation of the cervical vertebrae: association with various types of malocclusion. Braz Oral Res. 2012;26(2):145-50.

6. Kuc-Michalska M, Baccetti T. Duration of the pubertal peak in skeletal Class I and Class III subjects. Angle Orthod. 2010;80(1):54-7.

7. Salazar-Lazo R, Arriola-Guillen LE, Flores-Mir C. Duration of the peak of adolescent growth spurt in class I and II malocclusion subjects using a cervical vertebrae maturation analysis. Acta Odontol Latinoam. 2014;27:96-101.

8. García-Drago AG, Arriola-Guillén LE. Duration of the peak of growth in Class I and III subjects using the Baccetti's cervical vertebrae maturation analysis on lateral cephalometric radiographs. Oral Health Dent Management. 2014;13(4):963-66.

9. Jeelani W, Fida M, Shaikh A. The duration of pubertal growth peak among three skeletal classes. Dental Press J Orthod. 2016;21(5):67-74.

10. Perinetti G, Rosso L, Riatti R, Contardo L. Sagittal and Vertical Craniofacial Growth Pattern and Timing of Circumpubertal Skeletal Maturation: A Multiple Regression Study. Biomed Res Int. 2016;2016:1728712.

11. Lamparski DG. Skeletal age assessment utilizing cervical vertebrae [Dissertation]. Pittsburgh, PA: The University of Pittsburgh; 1972.

12. Beit P, Peltomaki T, Schatzle M, Signorelli L, Patcas R. Evaluating the agreement of skeletal age assessment based on hand-wrist and cervical vertebrae radiography. Am J Orthod Dentofacial Orthop. 2013;144(6):838-47.

13. Franchi L, Baccetti T, McNamara JA, Jr. Mandibular growth as related to cervical vertebral maturation and body height. Am J Orthod Dentofacial Orthop. 2000;118(3):335-40.

14. Mellion ZJ, Behrents RG, Johnston LE, Jr. The pattern of facial skeletal growth and its relationship to various common indexes of maturation. Am J Orthod Dentofacial Orthop. 2013;143(6):845-54.

15. Uysal T, Ramoglu SI, Basciftci FA, Sari Z. Chronologic age and skeletal maturation of the cervical vertebrae and hand-wrist: is there a relationship? Am J Orthod Dentofacial Orthop. 2006;130(5):622-8.

16. Ball G, Woodside D, Tompson B, Hunter WS, Posluns J. Relationship between cervical vertebral maturation and mandibular growth. Am J Orthod Dentofacial Orthop. 2011;139(5):e455-61.

17. Gu Y, McNamara JA, Jr. Mandibular growth changes and cervical vertebral maturation. a cephalometric implant study. Angle Orthod. 2007;77(6):947-53.

18. Gray S, Bennani H, Kieser JA, Farella M. Morphometric analysis of cervical vertebrae in relation to mandibular growth. Am J Orthod Dentofacial Orthop. 2016;149(1):92-8.

19. Nestman TS, Marshall SD, Qian F, Holton N, Franciscus RG, Southard TE. Cervical vertebrae maturation method morphologic criteria: Poor reproducibility. Am J Orthod Dentofacial Orthop. 2011;140(2):182-8.

20. Fishman LS. Radiographic evaluation of skeletal maturation. A clinically oriented method based on hand-wrist films. Angle Orthod. 1982;52(2):88-112.
21. Perinetti G, Sbardella V, Contardo L. Diagnostic reliability of the third finger middle phalanx maturation (MPM) method in the identification of the mandibular growth peak. Eur J Orthod. 2017;39(2):194-201.

22. Houston WJ. Relationships between skeletal maturity estimated from hand-wrist radiographs and the timing of the adolescent growth spurt. Eur J Orthod. 1980;2(2):81-93.

23. World Medical Association. WMA Declaration of Helsinki - Ethical Principles for Medical Research Involving Human Subjects; 2008.

24. Perillo L, Femiano A, Palumbo S, Contardo L, Perinetti G. Skeletal and dental effects produced by functional regulator-2 in pre-pubertal class II patients: a controlled study. Prog Orthod. 2013;14:1-18.

25. Springate SD. The effect of sample size and bias on the reliability of estimates of error: a comparative study of Dahlberg's formula. Eur J Orthod. 2012;34(2):158-63.

26. Landis JR, Koch GG. The measurement of observer agreement for categorical data. Biometrics. 1977;33(1):159-74.

27. Perinetti G. StaTips Part I: Choosing statistical test when dealing with differences. South Eur J Orthod Dentofac Res. 2016;3(1):4-5.

28. Perinetti G, Primozic J, Franchi L, Contardo L. Treatment effects of removable functional appliances in pre-pubertal and pubertal Class II patients: A systematic review and meta-analysis of controlled studies. PLoS One. 2015;10:1-35 e0141198.

29. Perinetti G, Primozic J, Furlani G, Franchi L, Contardo L. Treatment effects of fixed functional appliances alone or in combination with multibracket appliances: A systematic review and meta-analysis. Angle Orthod. 2014;85(3):480-92.

30. Baccetti T, Franchi L, Schulz SO, McNamara JA, Jr. Treatment timing for an orthopedic approach to patients with increased vertical dimension. Am J Orthod Dentofacial Orthop. 2008;133(1):58-64.

31. Baccetti T, Franchi L, Giuntini V, Masucci C, Vangelisti A, Defraia E. Early vs late orthodontic treatment of deepbite: a prospective clinical trial in growing subjects. Am J Orthod Dentofacial Orthop. 2012;142(1):75-82.

32. Ehsani S, Nebbe B, Normando D, Lagravere MO, Flores-Mir C. Shortterm treatment effects produced by the Twin-block appliance: a systematic review and meta-analysis. Eur J Orthod. 2015;37(2):170-6.

33. Marsico E, Gatto E, Burrascano M, Matarese G, Cordasco G. Effectiveness of orthodontic treatment with functional appliances on mandibular growth in the short term. Am J Orthod Dentofacial Orthop. 2011;139(1):24-36.

34. Thiruvenkatachari B, Harrison JE, Worthington HV, O'Brien KD. Orthodontic treatment for prominent upper front teeth (Class II malocclusion) in children. Cochrane Database Syst Rev. 2013:CD003452.

35. Perinetti G, Bianchet A, Franchi L, Contardo L. Cervical vertebral maturation: An objective and transparent code staging system applied to a 6-year longitudinal investigation. Am J Orthod Dentofacial Orthop. 2017;151(5):898-906.

36. Santiago RC, de Miranda Costa LF, Vitral RW, Fraga MR, Bolognese AM, Maia LC. Cervical vertebral maturation as a biologic indicator of skeletal maturity. Angle Orthod. $2012 ; 82(6): 1123-31$.

37. Hägg U, Taranger J. Maturation indicators and the pubertal growth spurt. Am J Orthod. 1982;82(4):299-309.

38. Grave KC, Brown T. Skeletal ossification and the adolescent growth spurt. Am J Orthod. 1976;69(6):611-9.

39. Parent AS, Teilmann G, Juul A, Skakkebaek NE, Toppari J, Bourguignon JP. The timing of normal puberty and the age limits of sexual precocity: variations around the world, secular trends, and changes after migration. Endocr Rev. 2003;24(5):668-93.

40. Karlberg J. Secular trends in pubertal development. Horm Res. 2002;57 Suppl 2:19-30. 